\title{
Recent (2018-2020) development in capillary electrophoresis
}

\author{
Ziting Gao ${ }^{1} \cdot$ Wenwan Zhong ${ }^{1}$ \\ Received: 4 February 2021 / Revised: 8 March 2021 / Accepted: 11 March 2021 / Published online: 22 March 2021 \\ (C) Springer-Verlag GmbH Germany, part of Springer Nature 2021
}

\begin{abstract}
Development of new capillary electrophoresis (CE) methodology and instrumentation, as well as application of CE to solve new problems, remains an active research area because of the attractive features of CE compared to other separation techniques. In this review, we focus on the representative works about sample preconcentration, separation media or capillary coating development, detector construction, and multidimensional separation in CE, which are judiciously selected from the papers published in 2018-2020.
\end{abstract}

Keywords Capillary electrophoresis $\cdot$ Preconcentration $\cdot$ Separation media and additives $\cdot$ Capillary coatings $\cdot$ Detectors

\section{Introduction}

Capillary electrophoresis (CE), even since its first introduction in 1981 [1], has been developed well into a mature and robust separation technique [2]. Compared to the traditional separation techniques like gel electrophoresis and liquid chromatography, CE carries the advantages of high simplicity in setup and miniaturization, fast separation with high resolution and efficiency, and low sample and solvent consumption. Thus, $\mathrm{CE}$ has been widely employed in biomedical research, forensic investigation, environmental monitoring, food quality examination [3], etc., to separate diverse analytes ranging from small ions, macromolecules, to microorganisms.

Continuous advancement of $\mathrm{CE}$ has been focused on overcoming the key obstacles in its operation, such as low concentration sensitivity and strong wall adsorption of compounds with large molecular mass or unfavorable charges [4], which could be particularly problematic when employed to analyze complex samples with high amounts of interfering compounds in the matrix. Modification of the separation condition with new column coating or buffer additives, fabrication of new separation setup to facilitate multidimensional separation, and development of preconcentration methods and detection systems have been actively explored $[5,6]$. Additionally, CE

Published in the topical collection celebrating ABCs 20th Anniversary.

Wenwan Zhong

wenwan.zhong@ucr.edu

1 Department of Chemistry, University of California-Riverside, 900 University Ave., Riverside, CA 92521, USA can be coupled with bioassays, such as enzyme assays [7] and systematic evolution of ligands by exponential enrichment (SELEX) [2], to expand its application scope. Herein, this review provides an overview of such developments in $\mathrm{CE}$ in the years of 2018-2020. For the works published before 2018, many elegant reviews can be found [2, 4-6]. Notably, exciting $\mathrm{CE}$ applications in analysis of proteins, pharmaceuticals, nucleic acids, metabolites, and carbohydrates, have been thoroughly discussed in a nice review recently published [8], which also provides an outlook for future directions towards improving the analysis of single cells and bio-particles. Thus, in this review, we mainly covered emerging and evolving advances in the aspects of sample preconcentration, separation media or coating development, detector construction, and multidimensional separation.

\section{Sample preconcentration}

The small column dimension determines that standard CE can only take in a very small volume of the sample, which greatly limits the overall detection sensitivity. Therefore, many online and off-line sample preconcentration methods have been developed to utilize electric field, phase distribution, flowgate, evaporation, etc., to improve analyte detectability [2].

\section{Electrophoretic preconcentration}

Electrophoretic preconcentration is the most common way for sample preconcentration in CE, because it is easy to be implemented without any change to the CE system. Primarily, it 
manipulates ion migration by controlling the direction of the electroosmotic flow (EOF) and analyte's electrophoretic mobility, as well as the electric field strength difference between the sample plug and the background electrolytes. A few examples include field-amplified sample stacking/injection (FASS/FASI), large-volume sample stacking/injection (LVSS/LVSI), sweeping, electrokinetic supercharging (EKS), transient isotachophoresis (tITP), isoelectric focusing (IEF), dynamic $\mathrm{pH}$ junction, and micelle to sample stacking (MSS).

Still, it could be challenging to implement electrophoretic preconcentration in some operations. For example, interfacing EKS to CE-MS requires dedicated design, including volatile buffer selection, flow controlling, and careful interface assembly. A new approach of EKS-based CE-MS method was described by using low-pH BGE to suppress EOF and facilitate positively charged analyte ionization during ESI [9]. Fifty millimolar ammonium formate at $\mathrm{pH} 2.5$ in the water $/ \mathrm{MeOH}$ $(60: 40 \mathrm{v} / \mathrm{v})$ was chosen for long injection with the aid of a leading electrolyte and terminating electrolyte. The separation sensitivity was enhanced by 5000 -fold over conventional injection methods. This coupling was employed for separation of biogenic amines from rat brain stem and whole Drosophila tissue by CE-ESI-MS/MS. Alternatively, CE separation with dynamic $\mathrm{pH}$ barrage junction focusing is well compatible with mass spectrometric detection, offering low sample consumption and straightforward optimization. Such focusing is generally utilized for analysis of peptides and intact proteins by CE-MS, and achieved by generating a pH-induced electrophoretic mobility variation at the sample/pH barrage boundary. It has been successfully applied for the study of the MCF7 breast cancer cell proteome using the bottom-up approach [10], and for top-down proteomic analysis of the zebrafish brain cerebellum $(\mathrm{Cb})$ and optic tectum (Teo) regions [11]. Also, quantitative analysis of bisphosphonate compounds [12] and digested monoclonal antibodies [13] was achieved by this technique.

In addition, electrophoretic focusing can be hyphenated with other preconcentration methods to further improve the enrichment effect. For instance, on-line hyphenation of FASI and tITP was developed by $\mathrm{Xu}$ et al. [14], and successful separation of sotalol and metoprolol in human urine was achieved within 10 min using phosphate as both the background and leading electrolyte, and glycine as the terminating electrolyte, with the limit of detection (LOD) and limit of quantitation (LOQ) for both compounds lower than $25 \mathrm{ng} /$ $\mathrm{mL}$. tITP was also coupled with LVSS by Kawai and coauthors to provide high salt tolerance to up to $10 \mathrm{mM} \mathrm{NaCl}$ in CE without losing resolution [15]. With the detection sensitivity enhanced by 2300 folds compared to the separation with normal injection, this method permitted profiling of the $\mathrm{N}$-glycans in approximately 100 cells. Moreover, a dual stacking method coupling sweeping and MSS [16] achieved around 43-77 folds improvement in LOD for the organic anions in Angelica sinensis, with the recovery ranging from 94.4 to $108.4 \%$.

New electrophoretic focusing techniques have been developed as well. To analyze bacteria in biological samples, Horká et al. etched a part of the fused silica capillary with supercritical water for static or dynamic adhesion of the bacteria cells [17]. The cells were then desorbed and preconcentrated by transient isotachophoretic stacking, subsequently separated by micelle electrokinetic chromatography (MEKC) with a non-inorganic surfactant. Such a design led to a high recovery of $83 \%$ that was independent of the sample matrix, and low LODs for bacteria in the physiological saline solution, bovine serum, or human blood, which were around $10^{2}-10^{3}$ cells $\mathrm{mL}^{-1}$.

\section{Chromatographic preconcentration}

Chromatographic extraction, like solid-phase extraction (SPE), single-drop microextraction (SDME), three-phase microelectroextraction (3PEE), and electromembrane extraction (EME), can isolate and enrich the molecules having strong interaction with the extraction media from complex matrices, which can then be eluted for $\mathrm{CE}$ analysis.

New extraction materials have been developed to reduce sample consumption, decrease operating cost, and shorten analysis time. Wu et al. fabricated the magnetic particles coated by the copolymer consisting of poly(3,4dihydroxyphenylalanine) and polyethyleneimine, and used them as the sorbents for off-line SPME of racemic catecholamines [18], which were then eluted and analyzed by FASICE. The magnetic particle-based SPME improved the detection sensitivity of the catecholamine enantiomers by 10 folds. Two novel periodic mesoporous organosilica materials have also been used as the sorbents for the extraction of 7 chiral drugs [19]. The good cleanup effect led to fast CE separation with high resolution reaching 2.4 8.5.

Off-line SPE systems contain no direct connection between the enrichment and separation devices, and are straightforward. To eliminate manual sample handling and increase reproducibility as well as sample recovery, on-line SPE-CE systems have been developed. Pont et al. investigated the performance of the gold nanoparticles coated by polymeric monoliths (AuNP@monoliths) as the microcartridges for protein analysis in SPE-CE-mass spectrometry (SPE-CE-MS) [20]. A 7-mm microcartridge was prepared by modifying the glycidyl methacrylate (GMA)-based monolithic capillary column with ammonia for attachment of AuNPs. The microcartridge can be simply used in a "plug-and-play" manner, providing high CE separating performance for analysis of human transthyretin with an LOD 50 times lower than that obtained by the traditional CE-MS method. 
A promising extraction approach, hollow fiber liquid-phase microextraction (HF-LPME), was introduced in 1999 for preconcentration and extraction of pharmaceuticals prior to CE identification and determination [21]. It is a "green" microextraction technology that is highly compatible with CE because of its enhanced solvent stability, improved selectivity, and lack of carry-over and memory effects between each extraction [22]. Especially, hollow fiber liquid-liquidliquid microextraction (HF-LLLME) has been reported to extract polar or ionizable compounds [23]. This method can also handle very complex matrices, as demonstrated by successful determination of basic drugs in physiological solutions, urine, and dried blood spot (DBS) samples [24].

Affinity-based extraction media have been employed to improve the specificity in target enrichment. Pero-Gascon et al. described an on-line aptamer-based affinity SPE as the pre-concentrator for CE-MS analysis of $\alpha$-synuclein ( $\alpha$-syn) in blood (Fig. 1) [25]. Under the optimal separation conditions, an LOD of $0.2 \mu \mathrm{g} \cdot \mathrm{mL}^{-1}$ was reached with excellent repeatability and microcartridge lifetime, and the method was proved to be effective for analysis of $\alpha$-syn in the lysates of red blood cells collected from healthy controls and Parkinson's disease (PD) patients.

Coupling EME with CE is intuitive because it requires electric fields to assist analyte migration. To achieve a uniform three-dimensional (3D) electric field in EME, Tan and coauthors printed a 3D EME device using a ROVA 3D FDM printer [26]. The device consisted of a hemispherical electrode sample vial with a smaller hemispherical 3D-printed porous membrane sitting inside that allows for anions to migrate through. Its performance was evaluated for determination of anions in water, achieving low LODs around $0.16-0.18 \mu \mathrm{M}$ for $\mathrm{NO}_{3}{ }^{-}, \mathrm{ClO}_{4}{ }^{-}$, and $\mathrm{SO}_{4}{ }^{2-}$.

Electrophoretic and chromatographic preconcentration can be combined to further enhance $\mathrm{CE}$ analysis of trace analytes in complex samples. Sun et al. coupled a novel microextraction method to FASI-CE for the enrichment and quantification of two beta-blockers in human urine (Fig. 1) [27]. They constructed a tapered SPE column by narrowing the diameter of a $\sim 5-\mathrm{cm}$-long fused silica capillary from 530 to $20 \mu \mathrm{m}$, allowing for the packing of the $45-\mu \mathrm{m}$ sorbent particles. This design is much smaller than the conventional SPE columns, does not require a frit to retain the sorbent particles, and can be reused for at least 8 times. Coupled with FASI-CE, such a column achieved about 21- and 19-fold sensitivity enhancement for atenolol and metoprolol, respectively, compared to the traditional CE method.

Extraction coupled with electrophoretic stacking can be used in non-aqueous $\mathrm{CE}$ as well, which is particularly advantageous in CE-MS because of the high volatility and low conductivity of the background electrolytes. For instance, SDME was combined with LVSS by Kim et al. for analysis of organic contaminants in soil sample [28]. After on-line SDME, the large volume of the pentanol extract was enriched by LVSS and further analyzed by non-aqueous CE/MS. This method achieved 600-1300 folds enrichment for the anionic analytes, including pesticide and herbicide compounds in soil samples, and provided impressive LODs in the range of $0.4-0.8 \mathrm{ppb}$.

\section{Miscellaneous}

Analyte cleanup and preconcentration designs not relying on electrophoresis and chromatography have been reported as well. For example, a membraneless evaporation process with diminished pressure was proposed by Paluch et al., allowing for sample preconcentration in a continuous mode [29]. This novel system could be used for preconcentration with different starting sample volumes, which was employed to determine the content of $\mathrm{Zn}$ in both drinking and waste water using CEUV with an LOD of $0.025 \mathrm{mg} / \mathrm{L}$.

Microdialysis (MD) was combined on-line with $C E-C^{4} D$ for analysis of saccharides in dairy products and honey by Tůma et al. [30] A flow-gating interface (FGI) made from a polydimethylsiloxane (PDMS) cross-connector was designed and inserted between the MD tubing and the inlet of CE, enabling the introduction of microdialysate to the FGI and then to the separation capillary. The method allowed rapid monitoring of food quality with minimum sample pretreatment and low LOQs in the range of $2.3-7.3 \mathrm{mg} / \mathrm{L}$. In addition, to minimize dilution of the dialysate, a new type of FGI was developed, in which the sample was transferred into the air, not to the gating solution [31]. A drop of the dialysate was forced from the delivery capillary into the empty injection space by the air pump and hydrodynamically injected into the separation capillary. The design was successfully applied to determine the cations in yogurts by $C E-C^{4} \mathrm{D}$.

\section{Separation media and additives}

CE separates analytes by their charge-to-size ratio, and successful separation is strongly dependent on the selection of the proper separation solution that can produce large enough difference in the electrophoretic mobility of the analytes and maintain the optimal and stable EOF. Thus, enormous efforts in CE method development have been devoted to finding the suitable separation media and buffer additives for analysis of diverse compounds.

\section{Synthetic receptors as buffer additives}

One big group of molecules used as the BGE additives in CE is synthetic receptors. They can interact with their ligands specifically, enhancing ligand resolution, and have lower molecular weights than proteins, more compatible as BGE additives. The biggest success of 

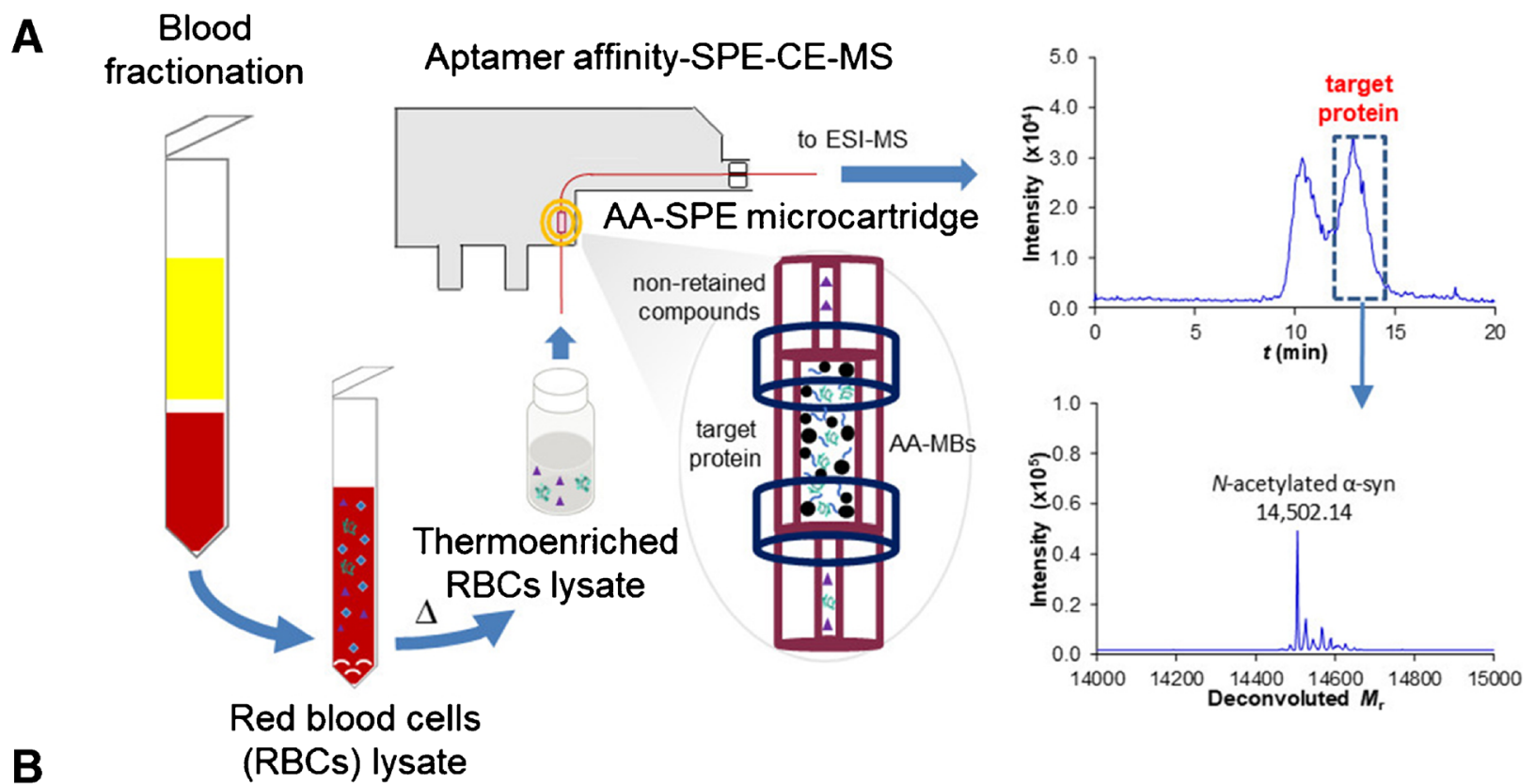

B (RBCs) lysate

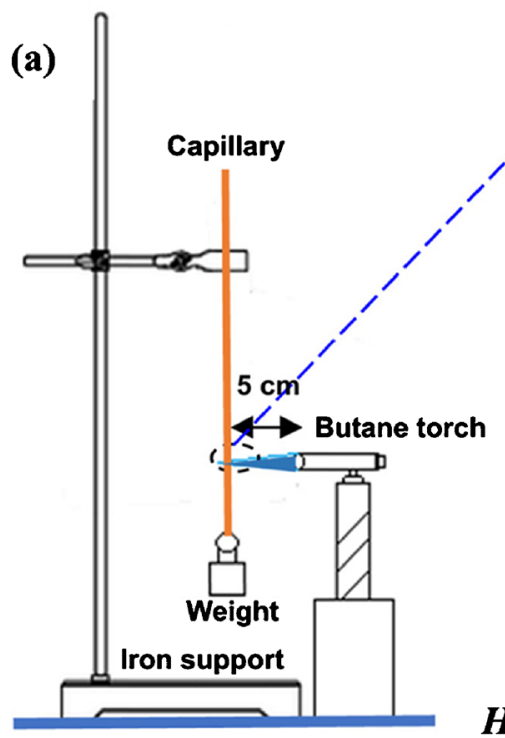

(b)



Empty tapered capillary

(c)





off-line

tCap- $\mu \mathrm{Ex}$

\section{Human urine samples}

tCap- $\mu \mathrm{Ex}$ column

(e)
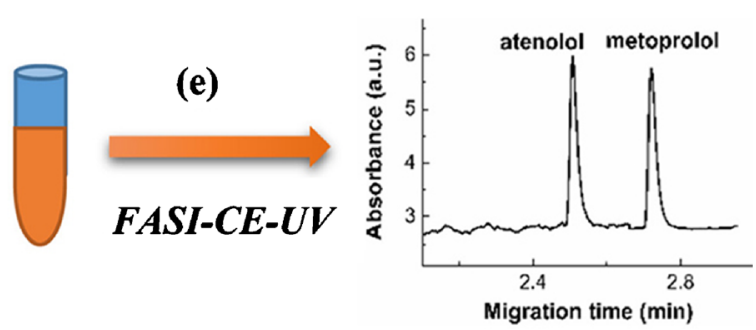

Fig. 1 Schematic illustration of on-line aptamer affinity SPE-CE-mass spectrometry for the analysis of blood $\alpha$-synuclein (A) and $t$ Cap- $\mu$ Ex approach coupled with FASI-CE for quantification of two $\beta$-blockers in human urine samples (B). For more information, please see [25, 27]

using synthetic receptors in CE separation is realized by the native and modified cyclodextrins (CDs), which excel at enantiomer separation [2].

Non-CD synthetic receptors have been employed as BGE additives in $\mathrm{CE}$ as well. Gamithromycin (Gam) was reported by Ren et al. to show good resolution of the chiral primary amines of primaquine (PMQ) and 1-aminoindan (AMI) [32], with N-methylformamide (NMF) as the non-aqueous solvent [33]. Our lab employed a group of non-CD receptors, tetrasulfonatocalix[4]arene (CX4), hexasulfonatocalix[6]arene (CX6), and cucurbit[7]uril (CB7), for analysis of methylation in peptides (Fig. 2) [34]. Peptides with different methylation levels at the same lysine position were separated using CX4 and CX6 in the BGE, which was further used to monitor the activity of the histone lysine demethylase JMJD2E. Later, this method was employed to analyze dual modifications, i.e., methylation and phosphorylation, on histone peptides using a linear polyacrylamide-coated capillary [35]. With the capability of separating the peptides carrying different modifications, the crosstalk between serine phosphorylation and lysine methylation was revealed.

Interestingly, synthetic receptors can be used to modify the surface of nanoparticles to enhance CE separation. CDmodified quantum dots (QDs) can act as both the pseudo- 

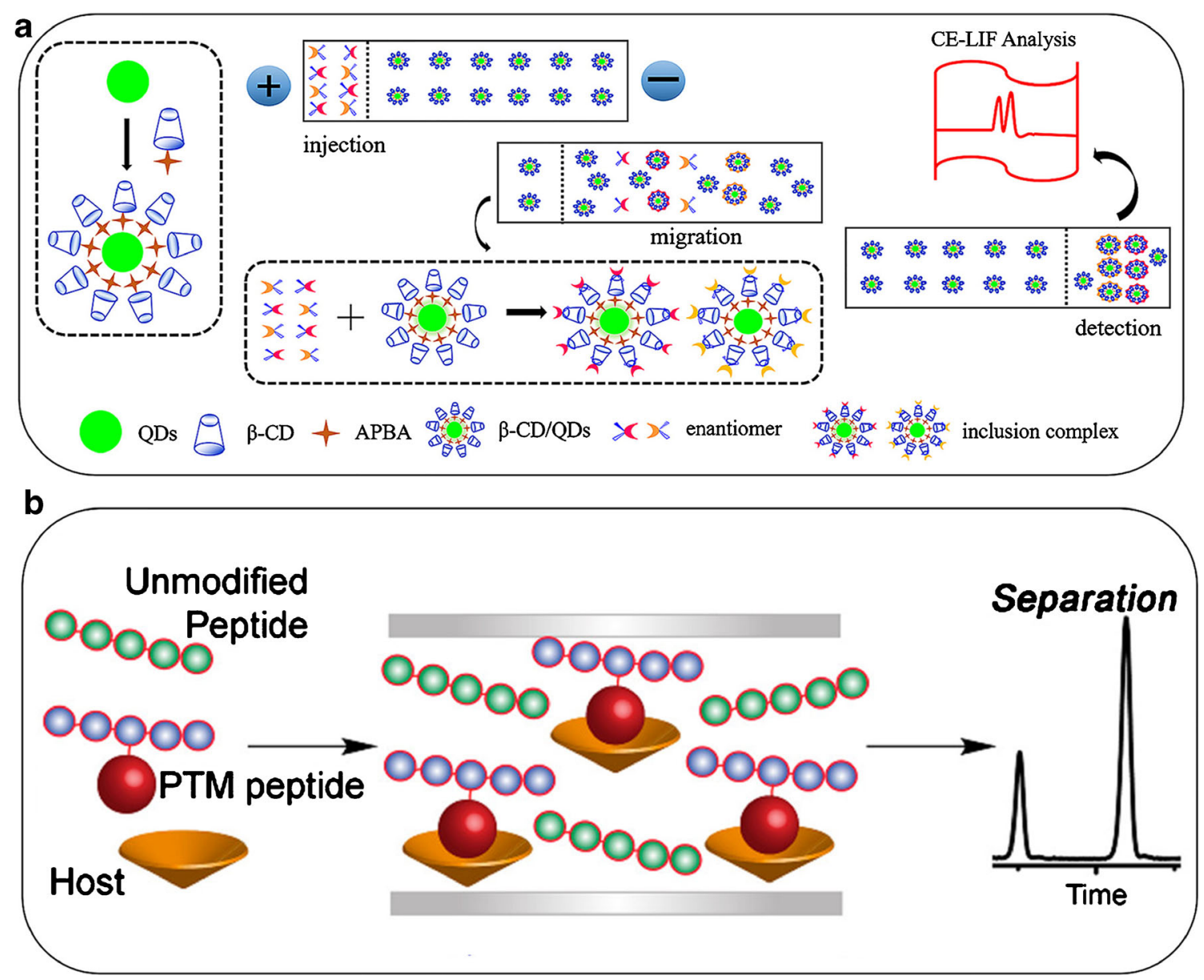

Fig. 2 Schematic illustration of $\beta$-cyclodextrin-modified quantum dots as pseudo-stationary phase for enantioseparation (A) and the host-assisted CE process with minimized structures of the host complexes (B). For more information, please see $[34,36]$

stationary phase to improve enantioseparation and the signaling tag for sensitive detection in CE-LIF without capillary pretreatment and analyte modification (Fig. 2) [36]. The RSDs of inter-day and intra-day repeatability varied in the range of $2.7-$ $8.1 \%$ for the peak area, $0.7-3.9 \%$ for the migration time, and $1.5-3.8 \%$ for the resolution. In addition, Liu et al. investigated the streptomycin-modified gold nanoparticles (ST-AuNPs) as an active chiral selector for enantioseparation of adrenaline, noradrenaline, and isoprenaline [37]. Under the optimized conditions, the racemic mixtures of the respective adrenergic drugs were separated within $7 \mathrm{~min}$ with a resolution of 7.5 and good inter-day and intra-day repeatability.

Using the receptors as BGE additives can also help to evaluate receptor-ligand binding. Owing to its high versatility and fast separation speed, CE is a powerful tool for study of noncovalent interactions. However, analyte detectability is often an issue, since adding the receptors to the BGE can increase background UV absorbance and reduce the signals from the analytes. The impurities in the samples could interfere with the measurement as well. Ligand derivatives or multiple detectors can be considered to overcome these difficulties [38].

\section{Ionic liquid as buffer additives}

Ionic liquids, because of their unique ionic and solvation capability, can serve as buffer additives as well. They can be added to MEKC as either modifiers or surfactants for improved separation efficiency and selectivity. Non-chiral ILs can be also used for enantiomer separation, as done by Xue et al., in which several tetraalkylammonium amino acid ILs (TAA-AAILs) were tested for CE enantioseparation of freeor dansyl-amino acids [39]. TAA-AAILs were also introduced to NACE with $\beta-C D$ as a chiral selector by Ren et al. [40] for separation of dansyl-amino acids (Dns-AA).

New chiral ILs have been developed in the past 2 years. For instance, a chiral IL, cholinium-clindamycin phosphate (Ch-CP), was developed and able to improve the separation of five racemic drugs, compared to clindamycin phosphate (CP) [41]. Noticeably, ILs can be mixed with other chiral selectors to produce a synergistic system for separation improvement. In one case, hydroxy acid-based chiral ionic liquids, namely tertramethylammonium- ${ }^{-}{ }^{-}$ pantothenate $\left(\mathrm{TMA}^{-}{ }^{-}-\mathrm{PAN}\right)$, tertramethylammonium- ${ }^{-}{ }^{-}$ 
quinate (TMA- $\left.{ }^{-}-\mathrm{QUI}\right)$, was designed and applied to establish a synergistic system with maltodextrin for CE enantioseparation by Yang et al. [42], significantly improving enantioselectivity. The other case involved the synthesis of three amino alcoholderived chiral ionic liquids, $N, N, N$-trimethyl- ${ }_{\mathrm{L}}$-valinolbis(trifluoromethanesulfon)imide, $N, N$-dimethyl- ${ }_{-}$-prolinolbis(trifluoromethanesulfon)imide, and $N, N, N$-trimethyl- ${ }_{\mathrm{L}^{-}}$ phenylalaninol-bis(trifluoromethanesulfon)imide), and their application for chiral separation when mixed with 2hydroxypropyl- $\beta$-cyclodextrin (HP- $\beta-C D)$ [43]. This optimized synergistic system significantly improved the separation of six drugs.

Even though ILs have attractive features, there are some arguments that once ILs are dissolved, they are no longer ILs, but just cations and anions [44]. Thus, further studies are required to uncover the specific mechanism of ILs with excellent separation performance.

\section{Organic solvents as separation media}

Although water is the most common solvent used in CE, alternative solvents are needed to provide sufficient solubility for special analytes, and enhance compatibility with mass spectrometry (MS)-based detection. Organic solvents provide good solubility to hydrophobic analytes and offer low surface tension and high volatility, making non-aqueous capillary electrophoresis (NACE) more suitable than aqueous CE for being coupled with ESI-MS [45]. Single or dual solvents can be used. For example, acetonitrile containing $25 \mathrm{mM}$ ammonium acetate and $100 \mathrm{mM}$ trifluoroacetic acid was used for separation of nine benzodiazepines in serum with a capillary coated by multiple ionic polymers [46]. The separation was completed at a negative voltage within $12 \mathrm{~min}$, with the LODs in MS reaching between 1.5 and $15.0 \mathrm{ng} / \mathrm{mL}$. A mixture of $20 \%$ acetonitrile and $78 \%$ methanol with $2 \%$ formic acid and $20 \mathrm{mM}$ ammonium formate was also employed for analysis of hydrophobic peptides [47], representing an effective alternative to the reversed-phase liquid chromatography (RPLC) for proteomic studies.

NACE offers several advantages over aqueous CE. For example, higher voltages can be used to provide fast analysis without increasing the risk of Joule heating. Additionally, by using different combinations of organic solvents or changing the proportion of the electrolytes, separation selectivity can be modified. Moreover, volatile solvents are more compatible with downstream MS detection. However, some concerns remain. There is lack of systematic investigations of solvent and electrolyte selection, and the correlation of the selection with separation selectivity. Besides, the presence of water in the electrophoresis medium may influence the separation efficiency.

\section{Capillary coatings and surface modifications}

When CE, especially CZE, is performed in a bare fused silica capillary, peak shape, resolution, and efficiency could be compromised by the interaction between samples and the inner wall of capillary. Analyte adsorption onto the capillary wall would change the wall conditions and thus affect the magnitude of EOF, leading to poor reproducibility in migration time. Analyte quantification would be strongly impacted by wall interaction as well, because the peak area would not be proportional to the injected amounts with some adsorbed on the wall. On the other hand, biological units can be immobilized on the capillary walls to conduct specific reactions prior to the separation, enabling investigations of enzymatic or cellular functions using CE. Thus, capillary coating and surface modification remain popular topics of $\mathrm{CE}$ research.

\section{Non-covalent and dynamic modifications}

Non-covalent, dynamic wall modification is typically the first choice researchers would explore to suppress analyte adsorption and modulate EOF, because it is simpler to be carried out and the coating material is easily exchangeable.

Jia et al. developed a dynamic modification method using the highly water-soluble methyl chitosan ( $\mathrm{MCh}$ ) polymer as the sieving matrix for protein separation. The charges of the amine groups could be tuned by adjusting the $\mathrm{pH}$ of $\mathrm{BGE}$, providing high flexibility in modulating the charge of the wall and in turn the magnitude of EOF [48]. This method showed great potential for protein glycoform analysis.

Nanomaterials can be employed for dynamic coating of capillary in CE as well. Geng et al. proposed a low-cost and effective capillary coating using zeolites imidazolate framework-8 (ZIF-8) [49]. The large surface area and the accessible tunnels and cages of ZIF-8 can benefit protein and chiral compound separation. By electrostatic interaction, ZIF-8 nanocrystals coated the inner surface of silica capillary, providing fast baseline separation of Lys, CC, BSA, and RNase A in 10 min with good reproducibility and stability. Additionally, when ${ }_{\text {L-glutamic acid was used }}$ as the selector ligand, $\mathrm{D}^{-}$and $\mathrm{L}_{\mathrm{L}}$-phenylalanine was successfully separated by the ZIF-8-coated capillary. In addition, carboxymethyl- $\beta$-cyclodextrin-functionalized magnetic microparticles (CD-MMPs) have been used to establish an open-tubular capillary electrochromatography system [50]. With an external magnetic field, coating by the CD-MMP was easy to realize, and the resultant capillary provided satisfactory repeatability and enantioselectivity for separation of propranolol, ofloxacin, amlodipine, chlortrimeton, tropicamide, and atenolol. 


\section{Covalent coatings}

Compared to dynamic coating, covalently attaching the coating material to the capillary wall can provide enhanced coating stability and separation reproducibility. There have been substantial improvements achieved lately to simplify the coating procedure and reduce the technical demand, using small molecules, polymers, etc.

A new capillary coating was introduced by linking Nacryloylamido ethoxyethanol (AAEE) to the silica surface via Si-C bonds. The coated capillary was employed for CEMS, and provided good $\mathrm{pH}$ stability and tolerance for harsh rinsing procedures (e.g., using strong acids, strong bases, and/ or organic solvents for rinsing) [51]. With the optimized coating method, outstanding performance for each coated capillary was found, including long-term storage at ambient conditions. Besides, the coating gave reproducible results for small molecules, peptides, and proteins that could be easily adsorbed to capillary surfaces.

Compared to surface modification by small molecules, polymer coating could be more stable and provide higher surface coverage. One photosensitive coating agent, diazotized poly (vinyl alcohol- $b$-styrene) (diazo-P(VA- $b$-St)), was developed [52] and exhibited enhanced stability and outstanding repeatability for protein separation. This coating had the capability to suppress protein adsorption on the inner surface of capillary, leading to good CE separation performance and repeatability.

A mixed polymer coating method has been developed as well, using poly(2-methyl-2-oxazoline) (PMOXA) and poly(acrylic acid) (PAA). The PMOXA-NH ${ }_{2}$ and PAA-SH were grafted on the bare fused silica capillary, and the coated capillary was used for protein adsorption and desorption under different separation conditions, which further applied for online extraction and preconcentration of lysozyme [53]. PMOXA/PAA-modified capillary exhibited an interesting behavior: surface adsorption was tunable by changing the $\mathrm{pH}$ and ionic strength of the running buffer. Lysozyme could be adsorbed onto the inner wall of capillary at $\mathrm{pH} 7$ while desorbed at $\mathrm{pH} 3$. The peak area of lysozyme was increased by 26 times compared to conventional CE separation, and the sensitivity was enhanced by $10^{5}$ folds.

Nanomaterials can be covalently attached to the capillary wall as well. Zhao et al. used graphene oxide (GO) as the coating reagent and DR as the coupling reagent for capillary coating to inhibit protein adsorption, following the same layer-by-layer self-assembly strategy mentioned above for DR and PS-PEO [54]. The GO-coated capillary showed great separation performance for proteins with excellent stability and reproducibility.

In a brief summary, on-covalent/dynamic capillary coatings are easier to apply and replenish, while covalent coatings can lead to higher stability and eliminate the possibility of interfering the detection system. However, more diverse coating materials should be developed to simplify the coating procedure and provide more functionality of the coated capillary. Additionally, developing automatic capillary coating approaches in commercial CE instruments can help reduce the technical barrier in preparation of coated capillaries on demand, attracting increasing interests.

\section{Wall immobilization of biological units}

Other than EOF modulation and elimination of analyte-wall interaction, biological entities, like proteins and cells, can be immobilized on the capillary wall, producing on-line microreactors to enable in situ reaction monitoring by $\mathrm{CE}$, which is highly useful in drug screening.

\section{Enzyme immobilization}

Enzymes can be immobilized onto the capillary wall by adsorption, cross-linking, encapsulation, and magnetic microparticles. The resultant immobilized enzyme microreactor (IMER) can realize analyte incubation, product separation, and detection in one capillary [55], providing a highly convenient system for rapid monitoring of enzyme activities. Various new immobilization methods have been reported in the past 2 years, aiming to ensure high enzyme activity and simplify immobilization process.

To enhance enzyme loading, nanomaterials can be used as the support, providing high relative surface areas for higher loading capacity of the enzyme molecules. One good example is to use the hydrophobic interaction and hydrogen bonding between GO and the negatively charged enzymes to facilitate CE-IMER [56]. In this method, poly (diallyldimethylammonium chloride) (PDDA) was firstly adsorbed onto the capillary wall to form a film and attract $\mathrm{GO}$ via electrostatic interaction. $\mathrm{L}$-lactate dehydrogenase $\left(\mathrm{L}_{-}-\mathrm{LDH}\right)$ was then immobilized on GO through hydrophobic interaction. The CE-IMER method exhibited repeatability with inter-day and batch-to-batch RSG smaller than 3.49 and $6.37 \%$, respectively.

Dopamine (DOPA) has been widely known for its unique feature in surface modification. It can self-assemble to form a polydopamine (PDA) coating which can be subsequently used for protein conjugation through non-covalent interactions, such as hydrophobic interaction and/or $\pi-\pi$ stacking. The same strategy has been applied to immobilize betaglucosidase (beta-Glu) on the capillary wall for CE-IMER [57]. The substrate of $\mathrm{p}$-nitrophenyl beta- $\mathrm{D}$-glucopyranoside and the inhibitor of castanospermine were used to demonstrate the system's suitability for enzyme kinetic and inhibition studies. Immobilization on GO via PDA provided good enzyme stability and assay repeatability: $89.5 \%$ of the initial immobilized beta-Glu activity remained after 50 continuous runs. 
One highly simple enzyme microreactor was constructed by Dadouch et al. [58]. They employed the strategy of transverse diffusion of laminar flow profiles (TDLFP) that relies on transverse diffusion to inject a plug of the reaction mixture and allow fast mixing inside the capillary and thus completion of the enzymatic reaction in a capillary. With the implementation of thermal control, fast enzymatic reaction and high-resolution separation were attained. Compared to off-line methodology, 1000-fold reduction of reactant consumption and 2-fold increase of the numbers of theoretical plates were observed.

Enzyme immobilization increases enzyme stability and reduces the experimental cost by avoiding the waste of enzyme. However, some concerns are associated with this approach. For example, when the incubation buffer and separation buffer are different, IMER is not suitable for enzymatic reactions. Moreover, immobilization operations could be complicated and hard to control, resulting in decreased enzyme activity [7].

\section{Cell capillary electrophoresis}

Cells are more suitable than enzymatic proteins to be used in drug screening and characterization by maintaining the natural conformation and bioactivity of proteins. Thus, immobilizing cells to the capillary wall and using them as the drug processor can benefit rapid drug screening and characterization [59]. A method called immobilized cell capillary electrophoresis (ICCE) has been introduced to screen the drugs targeting integrin macrophage antigen1 (MAC-1, CD11b/CD18) under optimal physiological conditions [60]. HEK293 cells that have overexpression in MAC-1-, CD11b-, or CD18 were immobilized on the capillary wall, and used to study their interaction with dimethylsulfoxide (DMSO; negative control) and lactosyl derivative Gu-4 (antagonist of MAC-1). Twenty-nine phenylethanoid glycosides from Cistanches Herba were screened qualitatively and the binding parameters of active compounds were calculated quantitatively. Also, better pharmaceutical efficacy and lower toxic side effects of the identified compounds were confirmed by pharmaceutical efficacy assays, proving the feasibility of ICCE in high-throughput screening for drug discovery.

Similar to proteins, cells can also be adsorbed to the capillary wall without covalent linkage, in which the cells can be put under approximately physiological conditions and maintain their maximum activities. Such a method was employed to use tumor cells/tissues as the interaction phase to allow rapid screening of multi-target antitumor drugs (Fig. 3) [61]. Compared to cell crosslinking, this method can renew the cells every run. However, the stability of the whole cells and inter-day repeatability based on different cell physiological status still need further investigation.

\section{Multidimentional separations}

For analysis of complex biological samples, one dimensional separation may not be sufficient. Thus, multidimensional CE has been developed to enhance resolution and separation performance.

One elegant work was conducted by Schlecht et al. to employ 2D CE for solving the issues of CE-MS coupling [62]. The first dimension using the mode of CZE, CIEF, MEKC, etc., was coupled with a UV detector and connected to CZE as the second dimension, which used volatile BGEs for high compatibility with MS. Successful separation and detection of intact monoclonal antibody (mAb) was achieved. Furthermore, their group developed MEKC-CZE-MS for analysis of protein impurities and fragments, and demonstrated the versatility of this system by analysis of the proteins extracted from soybeans [63].

However, coupling CE to other separation techniques is not entirely straightforward due to the ultralow sample volume and flow rate in CE, as well as the complicated design needed to connect the electric field-driven $\mathrm{CE}$ with the pressuredriven separation systems. Thus, off-line coupling is often employed. You et al. proposed the first example of off-line coupling of asymmetrical flow field flow fractionation (AF4) and capillary electrophoresis (CE) for separation of nanoparticles [64]. They studied the separation of a mixture of polystyrene nanoparticles (PS-NPs) that had different charge groups on the surface and comparable core sizes $(20,50$, and $100 \mathrm{~nm}$ ). With either method alone, low peak resolution and efficiency were observed, while the $2 \mathrm{D}$ off-line coupling of AF4 and CE system provided distinct regions for the mixtures having the core size of 20 and $50 \mathrm{~nm}$, although separation of the 100-nm particles remained not successful.

\section{Detectors}

\section{Mass spectrometry (MS)}

MS continues to be a powerful detector for CE. With the development of new and effective interfaces, CE can be coupled to many different MS systems to achieve high detection sensitivity.

Sasaki et al. used an ESI-MS adapter equipped with a grounded nebulizer for the regulation of electric currents at the interface between CE and the ESI source of HRMS [65]. This sheathless CE-MS coupling improved the detection sensitivity, and provided good repeatability and intermediate precision for analysis of 34 charged metabolites. Furthermore, they assessed their metabolome analysis platform using human-plasma extract and successfully detected 270 metabolites, proving the potential for large-scale screening of clinical samples for biomarker discovery. 


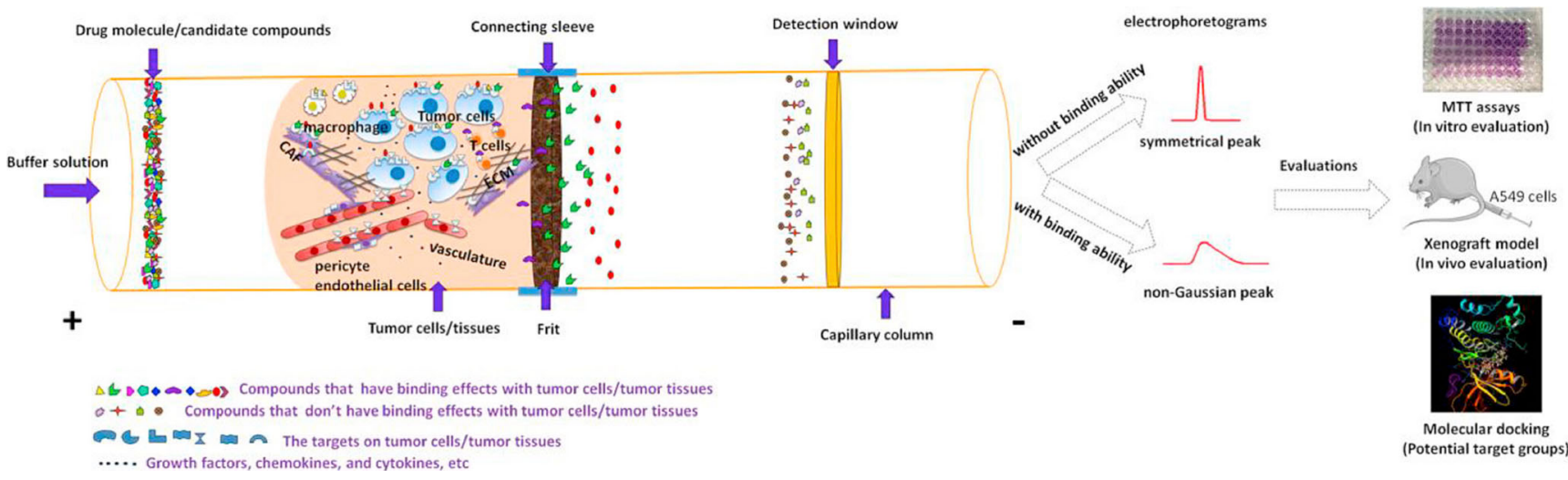

Fig. 3 Schematic overflow of non-immobilized tumor cells/tissues CE for rapid screening of multi-target antitumor drugs. For more information, please see [61]

A simple design was reported by Zhang et al. who placed a stainless steel needle at the capillary outlet for the nanoESI emitter and a reference electrode for the ESI spray to direct the liquid flow from the mobility capillary electrophoresis (MCE)

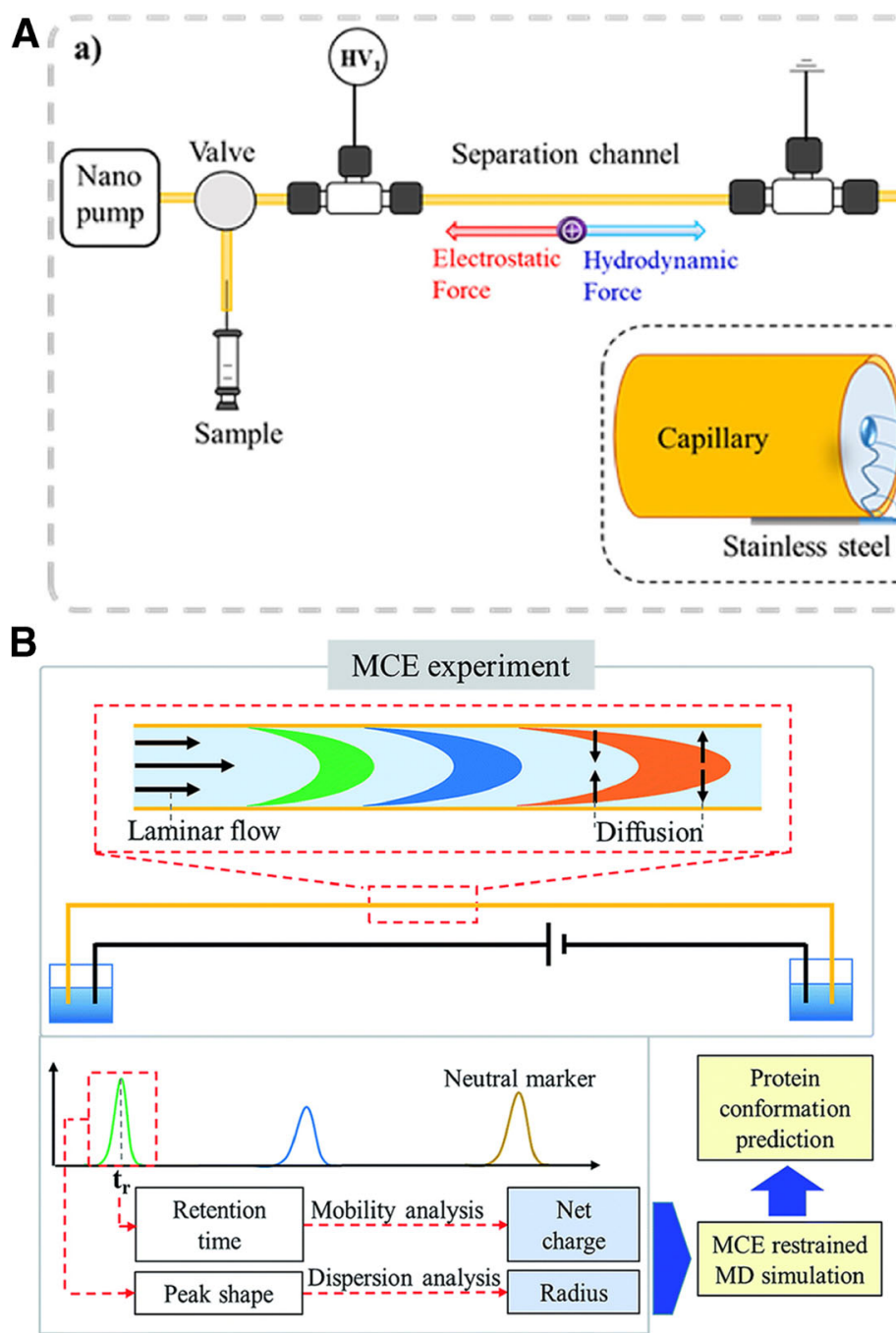

Fig. 4 (A) Schematic diagram of a new sheathless ionization interface. (a) The instrument setup with a zoom in view of the straight nanoESI interface. (b) A zoom in photo of the straight nanoESI. (c) A photo of the straight nanoESI MS interface. (B) Schematic diagram of the MCE-based

method for protein conformation and charge state analyses. (C) Scheme of the interface (top) and photographs of the liquid junction chip details. For more information, please see $[66,69,72]$ 
separation channel to the MS (Fig. 4) [66]. In MCE, a constant liquid flow replaces EOF in separation, and can be used to determine the effective radius of an ion, and when coupled with MS, to reveal the size of globular biomolecules [67]. Under optimized conditions, this design enhanced the ion signal intensity by $\sim 10$ times, compared to a commercial nanoESI source, with the LODs for peptides reaching $\sim 1 \mathrm{mg} / \mathrm{mL}$ to $\sim 20 \mu \mathrm{g} / \mathrm{mL}$. Similarly, a new medium throughput method was developed to couple MCE with native MS for protein analysis [68]. In this design, Tayler dispersion analysis (TDA) that provides the determination of the diffusion coefficient of analytes was integrated into MCE, allowing the measurement of ion separation, hydrodynamic radius, and effective charge in a single experiment (Fig. 4) [69]. In this work, the ion hydrodynamic radius and charge state distribution of 11 different globular proteins measured in the native MS were correlated to determine the shapes of these proteins, and monitor protein conformational changes, demonstrating feasibility in top-down proteomics and structural biology study regarding intact globular proteins and protein complexes.

To improve the conventional interfaces between CE and ICPMS, Holtkamp et al. designed a novel PTEE end-cap to convert a coaxial sheath-flow interface designed for coupling CE with ESIMS to an effective interface between CE and ICP-MS [70]. After optimizing the position of the capillary retracted inside the sprayer needle, and the flow rates of the sheath liquid and the carrier gas, the LOD for ${ }^{195} \mathrm{Pt}$ could reach $0.08 \mu \mathrm{M}$ with improved peak quality and sensitivity, compared to the commercially available CE-ICP-MS interface.

A mass spectrometry imaging (MSI) platform was built by coupling CE matrix-assisted laser desorption/ionization (MALDI) for improved coverage of neuropeptides [71]. In this method, the capillary was coated by polyethylenimine (PEI) to reduce peptide adsorption and LVSS was used to preconcentrate neuropeptides because of their low abundance in vivo. Such a continuous sample determination method detected a large number of neuropeptide with good reproducibility, demonstrating its ability to become an alternative for conventional LC-ESI-MS.

Computer modeling can help improve the interface design, as demonstrated by Krenkova et al. A hybrid liquid junctionbased interface was designed, guided by computer modeling, to include a liquid junction, a short transport channel, and a pointed sprayer intruding to the ESI source, and can be prepared by microfabrication technologies using plastic materials (Fig. 4) [72]. This novel system was applied to analyze a wide range of small peptides, proteins, and labeled oligosaccharides with low band broadening, high sensitivity, and good separation reproducibility.

IEF-ESI-MS has become a powerful method for accurate identification and quantification of proteoforms. Xu et al. [73] developed the automatic, on-line IEF-MS/MS platform using an electrokinetically pumped sheath flow CE-MS interface. In this platform, the inlet of linear polyacrylamide (LPA)-coated capillary was inserted into an acidic anolyte solution while its outlet was positioned into the interface filled with an acidic sheath buffer. After focusing, the $\mathrm{pH}$ gradient was gradually disrupted with protons migrating from the acidic anolyte and anions from the sheath buffer. Quantitative analysis of protein expression in the brains of male and female zebrafish was achieved for understanding the cellular processes.

Also, CE-MS is well suited for metabolic profiling, as exhibited by using CE-time-of-flight MS for quantification of hydrophilic metabolites in 247 subjects with different cancer stages [74]. Another good example is to employ multisegment injection-CE-MS for high-throughput metabolomic analysis of the placental with sex-specific differences [75]. Besides, some preconcentration methods was coupled to the CE-MS platform to reduce sample volume and detection limit, such as LLE for improved analysis of the metabolome of mice plasma [76] and FASI for enhanced study of the metabolome in individual neurons [77].

\section{Optical detection}

The small dimension of the capillary column determines that only a very small volume of the sample can be injected, and the optical path length is small, both contributing to the poor concentration sensitivity of on-column detection for CE [78], in particular, when coupled with optical detection methods, such as ultraviolet/visible (UV-Vis), fluorescence, and luminescence detection. Analyte labels that can give out intense signals, or improved detector design, have been two popular solutions to the detection problem of CE.

Graphene quantum dots (GQDs) with relatively uniform sizes and excellent photoluminescence properties were employed to enhance the emission of a fluoroquinolone for fluorimetric detection of ofloxacin in milk samples [79]. GQDs were injected into the capillary prior to the sample to selectively interact with ofloxacin so that it can be detected by fluorescence. Under the optimal CE conditions, LOD and LQD could reach 10.7 and $35.5 \mathrm{ng} / \mathrm{mL}$ respectively with a commercial $\mathrm{CE}$ device equipped with a multi-wavelength photoluminescence detector.

CE with UV-excited fluorescence detection for sensitive chiral analysis of amino acids (AAs) was studied by Prior et al. [80], employing optical fibers to direct the UV light from a Xe-Hg lamp and the emitted fluorescence to a chargecoupled device (CCD). LODs for enantioseparation of $\mathrm{D}^{-\mathrm{AA}}$ were in the 10-100-nM range. Furthermore, the system was applied to analyze cerebrospinal fluid (CSF) with the assistance of an improved sample cleanup step. An enhanced fluorescence detection system was equipped with a concave silver mirror, greatly increasing the sensitivity of light-emitting diode-induced fluorescence (LEDIF) [81]. The concave silver 
mirror reflected the emission of the target molecule with enhanced convergence, which was then detected by the photomultiplier tube (PMT), resulting in 16-fold increase in detection sensitivity compare to that achieved without the concave mirror. Two FITC-labeled tumor markers, ${ }_{L}$-Leu and ${ }_{\mathrm{L}}$-Val, were separated and detected in CE-LEDIF, with LODs reaching $150 \mathrm{nM}$. Additionally, an inexpensive and low-footprint silicon photomultiplier (SiPM) was used as a sensitive and affordable fluorescence detector [82]. SiPMs have a dynamic range that spans 5 orders of magnitude to detect down to $10^{-12} \mathrm{M}$ or $10^{-21} \mathrm{~mol}$ of fluorescein. It was proved to be able to provide high sensitivity for the measurement of enzyme activity in single cells. A miniature, portable, and sensitive instrumentation could be achieved by introducing the commercially available SiPMs.

A novel 3D-printed LED-induced fluorescence detector was designed with an integrated excitation source and optical filters for on-line, dual-point capillary detection by Casto et al. (Fig. 5) [83]. This 3D detector provided LODs of $613 \pm 13 \mathrm{pM}$ for fluorescein and was able to continuously monitor the conjugation of bovine serum albumin by fluorescein isothiocyanate.

In addition to fluorescence, chemiluminescence can be used for analyte detection in CE. A novel electrochemiluminescence (ECL) detector was designed to consist of silica sol-gel, zinc oxide nanoparticles (ZnO NPs), polyvinylpyrrolidone (PVP), and tris(2,2'-bipyridine) ruthenium (II) [84]. Under the optimized experimental conditions, LODs of quinapril hydrochloride $(\mathrm{QHCl})$ and its metabolite quinaprilat hydrochloride (QTHCl) were $3.6 \mathrm{ng} / \mathrm{mL}$ and $3.9 \mathrm{ng} / \mathrm{mL}$, respectively, with the BGE containing $14 \mathrm{mmol} / \mathrm{L}$ phosphate $(\mathrm{pH} 8.0)$ and 20\% n-propyl alcohol.

A

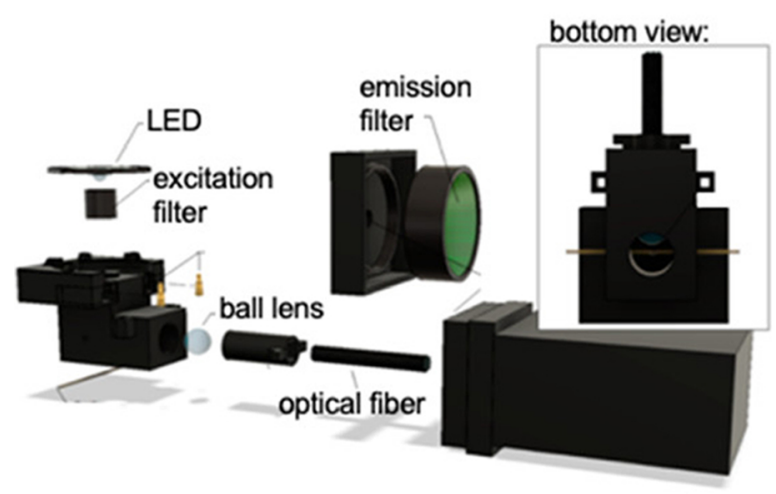

Fig. 5 Schematic diagram of a miniature 3D-printed LED-induced fluorescence detector (A) and a new 3D-printed cartridge that is used to improve temperature control of the capillary (B). The 3D-printed cartridge was basically composed of a part that was accommodated in the CE instrument (a) and another one that approaches the ESI source (b), in which the ESI probe (c) was held using a flexible O-ring (d). The silica

\section{Capacitively coupled contactless conductivity detection $\left(C^{4} D\right)$}

$\mathrm{C}^{4} \mathrm{D}$ has been widely used as a $\mathrm{CE}$ detector for quantification of both inorganic ions and organic compounds which could be applied in the field of forensic investigations and therapeutic developments.

To provide complementary information, $\mathrm{C}^{4} \mathrm{D}$ can be coupled with other detectors. For instance, it was coupled with UV detection to monitor meteorite bioleaching by Acidithiobacillus ferrooxidans [85] and thiol substrate hydrolysis during gold nanoparticle conjugation [86]. Coupled with MS and NACE separation, it was able to analyze food samples containing organic and inorganic compounds [87]. In this system, $C^{4} \mathrm{D}$ could detect inorganic ions and MS had low detection limits for organic biomolecules.

When coupling the low-selectivity $\mathrm{C}^{4} \mathrm{D}$ with the powerful MS, how to improve the CE separation performance of the thermally unstable compounds and how to stabilize the temperature control are two main problems. A new cartridge was thus constructed by $3 \mathrm{D}$ printing to improve temperature control in CE (Fig. 5) [88]. Up to two $\mathrm{C}^{4} \mathrm{D}$ devices could be accommodated inside the cartridge to form CE- $\left(C^{4} \mathrm{D}\right)^{2}$-ESI$\mathrm{MS} / \mathrm{MS}$ in an environment with proper dissipation of the thermal energy generated by Joule heating. Good peak shape and resolution, as well as reproducibility, were obtained for separation of monoethyl carbonate, a thermally unstable species.

Wang et al. optimized the structure of double-input (DI) $\mathrm{C}^{4} \mathrm{D}$, correcting the distortion in the peak shape and applied it for inorganic ion analysis by CE [89]. Compared to traditional $\mathrm{C}^{4} \mathrm{D}$, DIC ${ }^{4} \mathrm{D}$ exhibited lower baseline, higher sensitivity, lower noise, and good linear relationship.

\section{B}

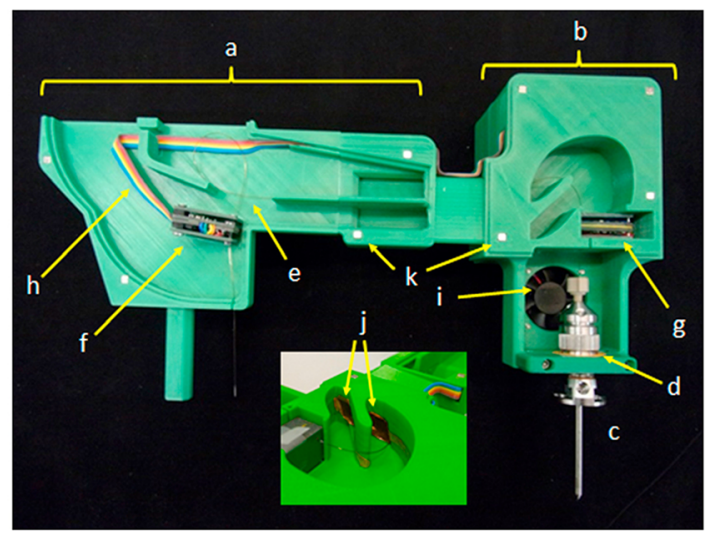

capillary (e) passed through the first (f) and the second $C^{4} \mathrm{D}(\mathrm{g})$. The electrical wiring (h) was connected to the microcontroller on the back of $b$. In the same compartment, there was an electrical connection for the fan (i). The copper foils (j) covered the inner walls of the ducts. Four-millimeter neodymium magnets $(\mathrm{k})$ were used to hold the covers of the compartments. For more information, please see [83, 88] 


\section{Miscellaneous}

Several new detection systems have also been developed during the period covered by this review. A remarkable one is to couple CE with surface plasmon resonance (SPR) through a microfluidic cell to achieve affinity assessment of antibodyantigen and enzyme-inhibitor binding in protein mixtures [90]. The SPR biosensor was used as the ground electrode.

When coupling CE with electrochemical detection, previous methods showed issues such as fragility or unavailability on the market. Hence, Kimlinger et al. presented a 3D-printed wall-jet electrode device to incorporate $\mathrm{CE}$ with electrochemical detection [91]. After separation, analytes were eluted by pressure, reducing the baseline noise compared to the traditional end-column detection. Separation of a mixture of catecholamines was realized by this system and the integration of a commercial CE device with the 3D electrode was demonstrated in a straightforward manner.

In addition, Nowak et al. demonstrated the proof of concept that microscale thermophoresis (MST) could be easily coupled to $\mathrm{CE}$ in an on-line flow system [92]. After electrophoretic separation, samples were directly introduced to the MST instrument in which they underwent thermophoretic analysis. During the measurement process, the EOF was temporally stopped. This system could be further applied to provide reliable data for measurement of ligand binding and molecular interactions, features unavailable for the current $\mathrm{CE}$ techniques. Furthermore, CE-MST coupled with MS is feasible by inserting the capillary outlet into the MS detector and using the MS-compatible buffers for thermophoretic analysis.

\section{Conclusion and outlooks}

$\mathrm{CE}$, although a matured separation technique, continues to evolve to benefit diverse analytical and bioanalytical studies, because of its versatile separation platforms and easy accommodation with separation media and additives. By no means can this review include all of the CE-related works published between 2018 and 2020. Instead, we selectively highlighted some unique developments representative to the major trend of advancement in CE methods and instrumentation, hoping to provide an up-to-date overview of the current state-of-theart development in the field.

With all these prior, elegant efforts to advance CE, we anticipate future works in CE methodology development will still be focused on improving analyte detection, tolerance to sample matrix, and analysis throughput in CE, because low tolerance for complex matrix components and low concentration sensitivity remain big challenges for $\mathrm{CE}$ applications. Thus, more convenient and effective sample cleanup, processing, and enrichment techniques are still in good demand. Methods and devices for enhancing CE coupling with the powerful and informative detectors, like MS, NMR, and even microscopy, will also be valuable in greatly expanding CE's applicability and helping to improve our understanding of the functions of the targets in interest. During or even after the COVID-19 pandemic, great attentions have been paid to the analysis of viral particles, and the development of vaccines and anti-viral drugs. The strong capability of $\mathrm{CE}$ in characterization of biologics, vaccines, and native vesicles [93] provides good premise for $\mathrm{CE}$ to make substantial impacts to such developments. Newly discovered analyte-interacting reagents or solvents, such as synthetic receptors, affinity probes, and ionic liquids, have been broadening the scope of analytes addressable by $\mathrm{CE}$. We anticipate more exploration of such reagents will be continued to develop CE solutions for challenging analytes [8].

Future development could further improve CE instrumentation as well to enhance the accessibility of CE platforms, and render cost-effective $\mathrm{CE}$ applications in resource-limited labs. For example, cheaper and purpose-made CE instruments have been developed for such purposes [9495]. CZE with an automatic fraction collector which holds microtiter plates and even Petri dishes were fabricated for successful determination of bacteria from a complex microbiome by downstream culturing and sequencing [96], and isolation of intact sperm cells for quantitative PCR analysis of male DNA [97]. Simplified and portable CE devices will continue to be developed with the advancement in $3 \mathrm{D}$ printing and microfabrication.

$\mathrm{CE}$ analysis of biological molecules has been nicely reviewed in 2020 [8]. Some noticeable, very recent works in this area include identification and quantification of glycan/ glycosylation accomplished through the development of new aminopyrene-based labeling tags [98]; new capillary coating materials like methyl chitosan [48]; and new preconcentration methods like on-line $\mathrm{TiO}_{2}$ extraction and off-line $\mathrm{Zr}^{4+}$-modified monolith metal through ion affinity chromatography (IMAC) [99100]. Single-cell analysis represents another important application area of $\mathrm{CE}$, because the small dimension of capillary columns fit well for manipulation of individual cells. We discussed the impressive determination of singlecell metabolomics in individual neurons above [77]. Apart from this, CE-enabled quantification of signaling molecules was demonstrated by detecting intra- and extracellular nitric oxide with the integration of single-cell injection, on-column derivatization, and cell lysis [101]. Microfluidic CE also provides the miniaturization needed for monitoring the behaviors of single cells. For example, a microfluidic device modeling in vivo environments was reported to facilitate investigation of cell-cell interactions [8]. CE provides excellent performance in clinical analysis as well [102], because it can deliver high separation power, is highly versatile in its implementation, and consume small sample amounts. For example, a portable CE analyzer was employed for detection of abused drugs in oral fluid [103], and HF-LLME or microwave-assisted extraction (MAE) was coupled with CE for drug analysis in dried blood 
spot (DBS) samples [24104]. No doubt CE will remain a highly effective tool for study of biomolecules in cells, tissues, or other biological samples, as well as for high-throughput analysis of drugs and their metabolites in body fluids. Worth noting, CE's capability in rapid screening of nucleic acids and proteins, and its high separation power for modified biomolecules, such as DNA adducts, protein post-translational modification, and noncoding RNA alkylation, will certainly make it an important tool for analysis of epigenome and epitranscriptome, to gain better understanding of how biological processes are regulated by such factors.

In summary, as an established analytical tool, CE will continue to evolve into a more advanced analytical tool and be adopted by experts beyond the field of separation to help solve problems in diverse areas.

Funding W. Zhong acknowledges the support from the National Science Foundation award (CHE-1707347).

\section{Declarations}

Conflict of interest The authors declare no competing interests.

\section{References}

1. Jorgenson JW, Lukacs KD. Zone electrophoresis in open-tubular glass capillaries. Anal Chem. 1981;53(8):1298-302.

2. Harstad RK, Johnson AC, Weisenberger MM, Bowser MT. Capillary electrophoresis. Anal Chem. 2016;88(1):299-319.

3. Piñero M-Y, Bauza R, Arce L. Thirty years of capillary electrophoresis in food analysis laboratories: potential applications. Electrophoresis. 2011;32(11):1379-93.

4. Timerbaev AR. Element speciation analysis using capillary electrophoresis: twenty years of development and applications. Chem Rev. 2013;113(1):778-812.

5. Ramos-Payán M, Ocaña-Gonzalez JA, Fernández-Torres RM, Llobera A, Bello-López MÁ. Recent trends in capillary electrophoresis for complex samples analysis: a review. Electrophoresis. 2018;39(1):111-25.

6. Voeten RLC, Ventouri IK, Haselberg R, Somsen GW. Capillary electrophoresis: trends and recent advances. Anal Chem. 2018;90(3):1464-81.

7. Cheng M, Chen Z. Recent advances in screening of enzymes inhibitors based on capillary electrophoresis. J Pharm Anal. 2018;8(4):226-33.

8. Kristoff CJ, Bwanali L, Veltri LM, Gautam GP, Rutto PK, Newton EO, et al. Challenging bioanalyses with capillary electrophoresis. Anal Chem. 2020;92(1):49-66.

9. Wells SS, Dawod M, Kennedy RT. CE-MS with electrokinetic supercharging and application to determination of neurotransmitters. Electrophoresis. 2019;40(22):2946-53.

10. Yang Z, Shen X, Chen D, Sun L. Microscale reversed-phase liquid chromatography/capillary zone electrophoresis-tandem mass spectrometry for deep and highly sensitive bottom-up proteomics: identification of 7500 proteins with five micrograms of an MCF7 proteome digest. Anal Chem. 2018;90(17):10479-86.

11. Lubeckyj RA, Basharat AR, Shen X, Liu X, Sun L. Large-scale qualitative and quantitative top-down proteomics using capillary zone electrophoresis-electrospray ionization-tandem mass spectrometry with nanograms of proteome samples. J Am Soc Mass Spectrom. 2019;30(8):1435-45.

12. Zhao T, Wang L, Li Y, Chen S, Wang R, Chen DDY. Quantification of the bisphosphonate alendronate using capillary electrophoresis mass spectrometry with dynamic $\mathrm{pH}$ barrage junction focusing. Electrophoresis. 2021;42(4):350-9.

13. Wang L, Cheng J, McNutt JE, Morin GB, Chen DDY. Dynamic $\mathrm{pH}$ barrage junction focusing of amino acids, peptides, and digested monoclonal antibodies in capillary electrophoresismass spectrometry. Electrophoresis. 2020;41(21-22):1832-42.

14. Xu L, Meng W, Lu J, Cui F, Gao L, Chen L, et al. Hyphenation of field-amplified sample injection and transient isotachophoresis in $\mathrm{CE}$ for the determination of sotalol and metoprolol in human urine samples. J Sep Sci. 2020;43(11):2193-200.

15. Kawai T, Ota N, Imasato A, Shirasaki Y, Otsuka K, Tanaka Y. Profiling of N-linked glycans from 100 cells by capillary electrophoresis with large-volume dual preconcentration by isotachophoresis and stacking. J Chromatogr A. 2018;1565:13844.

16. Yang X, Hao L, Zhang S, Wang C, Wang Z. Sweeping-micelle to solvent stacking for the on-line preconcentration and determination of organic acids in Angelica sinensis by capillary electrophoresis. RSC Adv. 2018;8(15):7949-55.

17. Horká M, Šalplachta J, Karásek P, Rủžička F, Roth M. Online concentration of bacteria from tens of microliter sample volumes in roughened fused silica capillary with subsequent analysis by capillary electrophoresis and matrix-assisted laser desorption/ ionization time-of-flight mass spectrometry. ACS Infectious Diseases. 2020;6(3):355-65.

18. Wu J, Li Z, Jia L. Solid phase extraction and capillary electrophoretic separation of racemic catecholamines by using magnetic particles coated with a copolymer prepared from poly $(3,4-$ dihydroxyphenylalanine) and polyethyleneimine. Microchim Acta. 2019;186(9):627.

19. Valimaña-Traverso J, Morante-Zarcero S, Pérez-Quintanilla D, García MÁ, Sierra I, Marina ML. Periodic mesoporous organosilica materials as sorbents for solid-phase extraction of drugs prior to simultaneous enantiomeric separation by capillary electrophoresis. J Chromatogr A. 2018;1566:135-45.

20. Pont L, Marin G, Vergara-Barberán M, Gagliardi LG, Sanz-Nebot V, Herrero-Martínez JM, et al. Polymeric monolithic microcartridges with gold nanoparticles for the analysis of protein biomarkers by on-line solid-phase extraction capillary electrophoresis-mass spectrometry. J Chromatogr A. 2020;461097.

21. Pedersen-Bjergaard S, Rasmussen KE. Liquid-liquid-liquid microextraction for sample preparation of biological fluids prior to capillary electrophoresis. Anal Chem. 1999;71(14):2650-6.

22. Filippou O, Bitas D, Samanidou V. Green approaches in sample preparation of bioanalytical samples prior to chromatographic analysis. J Chromatogr B. 2017;1043:44-62.

23. Kohler I, Schappler J, Rudaz S. Microextraction techniques combined with capillary electrophoresis in bioanalysis. Anal Bioanal Chem. 2013;405(1):125-41.

24. Miková B, Dvořák M, Ryšavá L, Kubán̆ P. Hollow fiber liquidphase microextraction at-line coupled to capillary electrophoresis for direct analysis of human body fluids. Anal Chem. 2020;92(10):7171-8.

25. Pero-Gascon R, Benavente F, Minic Z, Berezovski MV, SanzNebot V. On-line aptamer affinity solid-phase extraction capillary electrophoresis-mass spectrometry for the analysis of blood $\alpha$-synuclein. Anal Chem. 2020;92(1):1525-33.

26. Tan ML, Zhang M, Li F, Maya F, Breadmore MC. A threedimensional printed electromembrane extraction device for capillary electrophoresis. J Chromatogr A. 2019;1595:215-20. 
27. Sun S, Wang Y, Liu X, Fu R, Yang L. Rapid and sensitive tapered-capillary microextraction combined to on-line sample stacking-capillary electrophoresis for extraction and quantification of two beta-blockers in human urine. Talanta. 2018;180:90-7.

28. Kim J, Choi K, Chung DS. Synergistic coupling of in-line singledrop microextraction and on-line large-volume sample stacking for capillary electrophoresis/mass spectrometry. Anal Bioanal Chem. 2019;411(5):1067-73.

29. Paluch J, Kozak J, Wieczorek M, Wozniakiewicz M, Golab M, Poltorak E, et al. Novel approach to sample preconcentration by solvent evaporation in flow analysis. Molecules. 2020;25(8).

30. Tůma P, Sommerová B, Daněček V. On-line coupling of capillary electrophoresis with microdialysis for determining saccharides in dairy products and honey. Food Chem. 2020;316:126362.

31. Opekar F, Hraníček J, Tůma P. Rapid determination of majority cations in yoghurts using on-line connection of capillary electrophoresis with mini-dialysis. Food Chem. 2020;308:125647.

32. Ren S, Zhang Q, Xue S, Liu S, Rui M. Use of gamithromycin as a chiral selector in capillary electrophoresis. J Chromatogr A. 1624;2020:461099.

33. Yang X, Yan Z, Yu T, Du Y, Chen J, Liu Z, et al. Study of the enantioselectivity and recognition mechanism of chiral dual system based on chondroitin sulfate D in capillary electrophoresis. Anal Bioanal Chem. 2018;410(23):5889-98.

34. Lee J, Perez L, Liu Y, Wang H, Hooley RJ, Zhong W. Separation of methylated histone peptides via host-assisted capillary electrophoresis. Anal Chem. 2018;90(3):1881-8.

35. Lee J, Chen J, Sarkar P, Xue M, Hooley RJ, Zhong W. Monitoring the crosstalk between methylation and phosphorylation on histone peptides with host-assisted capillary electrophoresis. Anal Bioanal Chem. 2020;412(24):6189-98.

36. Wang T, Cheng Y, Zhang Y, Zha J, Ye J, Chu Q, et al. $\beta$ Cyclodextrin modified quantum dots as pseudo-stationary phase for direct enantioseparation based on capillary electrophoresis with laser-induced fluorescence detection. Talanta. 2020;210: 120629.

37. Liu C, Zhang J, Zhang X, Zhao L, Li S. Enantiomeric separation of adrenaline, noradrenaline, and isoprenaline by capillary electrophoresis using streptomycin-modified gold nanoparticles. Microchim Acta. 2018;185(4):227.

38. Fanali S, Chankvetadze B. Some thoughts about enantioseparations in capillary electrophoresis. Electrophoresis. 2019;40(18-19):2420-37.

39. Xue S, Ren S, Wang L, Zhang Q. Evaluation of tetraalkylammonium amino acid ionic liquids as chiral ligands in ligand-exchange capillary electrophoresis. J Chromatogr A. 1611;2020:460579.

40. Ren S, Xue S, Sun X, Rui M, Wang L, Zhang Q. Investigation of the synergistic effect of chiral ionic liquids as additives in nonaqueous capillary electrophoresis for enantioseparation. J Chromatogr A. 1609;2020:460519.

41. Xu H, Du Y, Feng Z, Sun X, Liu J. Synthesis of a chiral ionic liquid, cholinium-clindamycin phosphate, as sole chiral selector in capillary electrophoresis. J Chromatogr A. 1615;2020:460721.

42. Yang X, Du Y, Feng Z, Liu Z, Li J. Establishment and molecular modeling study of maltodextrin-based synergistic enantioseparation systems with two new hydroxy acid chiral ionic liquids as additives in capillary electrophoresis. J Chromatogr A. 2018;1559:170-7.

43. Ma X, Du Y, Sun X, Liu J, Huang Z. Synthesis and application of amino alcohol-derived chiral ionic liquids, as additives for enantioseparation in capillary electrophoresis. J Chromatogr A. 1601;2019:340-9.

44. Zhang Q. Ionic liquids in capillary electrophoresis for enantioseparation. Trends Anal Chem. 2018;100:145-54.
45. Scriba GKE. Nonaqueous capillary electrophoresis-mass spectrometry. J Chromatogr A. 2007;1159(1):28-41.

46. Švidrnoch M, Boráňová B, Tomková J, Ondra P, Maier V. Simultaneous determination of designer benzodiazepines in human serum using non-aqueous capillary electrophoresis - tandem mass spectrometry with successive multiple ionic - polymer layer coated capillary. Talanta. 2018;176:69-76.

47. Cheng J, Chen DDY. Nonaqueous capillary electrophoresis mass spectrometry method for determining highly hydrophobic peptides. Electrophoresis. 2018;39(9-10):1216-21.

48. Jia Y, Cao J, Zhou J, Zhou P. Methyl chitosan coating for glycoform analysis of glycoproteins by capillary electrophoresis. Electrophoresis. 2020;41(9):729-34.

49. Geng Z, Song Q, Yu B, Cong H. Using ZIF-8 as stationary phase for capillary electrophoresis separation of proteins. Talanta. 2018;188:493-8.

50. Sun X, Guo J, Yu T, Du Y, Feng Z, Zhao S, et al. A novel coating method for CE capillary using carboxymethyl- $\beta$-cyclodextrinmodified magnetic microparticles as stationary for electrochromatography enantioseparation. Anal Bioanal Chem. 2019;411(6):1193-202.

51. Meixner M, Pattky M, Huhn C. Novel approach for the synthesis of a neutral and covalently bound capillary coating for capillary electrophoresis-mass spectrometry made from highly polar and $\mathrm{pH}$-persistent $\mathrm{N}$-acryloylamido ethoxyethanol. Anal Bioanal Chem. 2020;412(3):561-75.

52. Yu B, Peng Q, Usman M, Ahmed A, Chen Y, Chen X, et al. Preparation of photosensitive diazotized poly (vinyl alcohol-b-styrene) covalent capillary coatings for capillary electrophoresis separation of proteins. J Chromatogr A. 2019;1593:174-82.

53. Hou M, Zhang M, Chen L, Gong K, Pan C, Wang Y. Amplification of lysozyme signal detected in capillary electrophoresis using mixed polymer brushes coating with switchable properties. Talanta. 2019;202:426-35.

54. Zhao X-Y, Wang M-H, Liu X-F, Cong H-L, Shen Y-Q, Yu B. Preparation of diazoresin/graphene oxide covalent coated capillary for separation of proteins by capillary electrophoresis. Ferroelectrics. 2019;546:74-84.

55. Wang X, Li K, Adams E, Schepdael AV. Recent advances in CEmediated microanalysis for enzyme study. Electrophoresis. 2014;35(1):119-27.

56. Li X, Yin Z, Cui X, Yang L. Capillary electrophoresis-integrated immobilized enzyme microreactor with graphene oxide as support: immobilization of negatively charged L-lactate dehydrogenase via hydrophobic interactions. Electrophoresis. 2020;41(3-4): 175-82.

57. Wu Z-Y, Zhang H, Li Q-Q, Yang F-Q, Li D-Q. Capillary electrophoresis-based online immobilized enzyme reactor for beta-glucosidase kinetics assays and inhibitors screening. J Chromatogr B. 2019;1110-1111:67-73.

58. Dadouch M, Ladner Y, Bich C, Larroque M, Larroque C, Morel J, et al. An in-line enzymatic microreactor for the middle-up analysis of monoclonal antibodies by capillary electrophoresis. Analyst. 2020;145(5):1759-67.

59. Yu HM, Tseng MJ, Fang JM, Phutrakul S, Chen ST. Capillary electrophoresis using immobilized whole cells with overexpressed endothelin receptor for specific ligand screening. Electrophoresis. 2004;25(7-8):1034-41.

60. Wu R, Li C, Sun X, Zhang S, Liang C, Jiang Y, et al. Rapid screening of anti-tumor metastasis drugs targeting integrin macrophage antigen-1 using immobilized cell capillary electrophoresis. Analyst. 2018;143(20):4981-9.

61. Wu R, Li C, Li C, Ren J, Sun X, Zhang S, et al. Rapid screening of multi-target antitumor drugs by nonimmobilized tumor cells/ tissues capillary electrophoresis. Anal Chim Acta. 2019;1045: $152-61$. 
62. Schlecht J, Jooß K, Neusüß C. Two-dimensional capillary electrophoresis-mass spectrometry (CE-CE-MS): coupling MSinterfering capillary electromigration methods with mass spectrometry. Anal Bioanal Chem. 2018;410(25):6353-9.

63. Römer J, Montealegre C, Schlecht J, Kiessig S, Moritz B, Neusüß C. Online mass spectrometry of CE (SDS)-separated proteins by two-dimensional capillary electrophoresis. Anal Bioanal Chem. 2019;411(27):7197-206.

64. You Z, Jakubowski N, Panne U, Weidner SM. Separation of polystyrene nanoparticles with different coatings using twodimensional off-line coupling of asymmetrical flow field flow fractionation and capillary electrophoresis. J Chromatogr A. 2019;1593:119-26.

65. Sasaki K, Sagawa H, Suzuki M, Yamamoto H, Tomita M, Soga T, et al. Metabolomics platform with capillary electrophoresis coupled with high-resolution mass spectrometry for plasma analysis. Anal Chem. 2019;91(2):1295-301.

66. Zhang F, Hong J, Xu W, Qu F. Straight nano-electrospray ionization and its coupling of mobility capillary electrophoresis to mass spectrometry. Talanta. 2020;206:120183.

67. He M, Luo P, Hong J, Wang X, Wu H, Zhang R, et al. Structural analysis of biomolecules through a combination of mobility capillary electrophoresis and mass spectrometry. ACS Omega. 2019;4(1):2377-86.

68. Wu H, Zhang R, Zhang W, Hong J, Xiang Y, Xu W. Rapid 3dimensional shape determination of globular proteins by mobility capillary electrophoresis and native mass spectrometry. Chem Sci. 2020;11(18):4758-65.

69. Zhang W, Wu H, Zhang R, Fang X, Xu W. Structure and effective charge characterization of proteins by a mobility capillary electrophoresis based method. Chem Sci. 2019;10(33):7779-87.

70. Holtkamp HU, Morrow SJ, Kubanik M, Hartinger CG. Hyphenation of capillary electrophoresis to inductively coupled plasma mass spectrometry with a modified coaxial sheath-flow interface. J Chromatogr A. 2018;1561:76-82.

71. DeLaney K, Li L. Capillary electrophoresis coupled to MALDI mass spectrometry imaging with large volume sample stacking injection for improved coverage of $\mathrm{C}$. borealis neuropeptidome. Analyst. 2020;145(1):61-9.

72. Krenkova J, Kleparnik K, Luksch J, Foret F. Microfabricated liquid junction hybrid capillary electrophoresis-mass spectrometry interface for fully automated operation. Electrophoresis. 2019;40(18-19):2263-70.

73. Xu T, Shen X, Yang Z, Chen D, Lubeckyj RA, McCool EN, et al. Automated Capillary Isoelectric Focusing-Tandem Mass Spectrometry for Qualitative and Quantitative Top-Down Proteomics. Anal Chem. 2020;92(24):15890-8.

74. Udo R, Katsumata K, Kuwabara H, Enomoto M, Ishizaki T, Sunamura M, et al. Urinary charged metabolite profiling of colorectal cancer using capillary electrophoresis-mass spectrometry. Sci Rep. 2020;10(1):21057.

75. Saoi M, Kennedy KM, Gohir W, Sloboda DM, Britz-McKibbin P. Placental metabolomics for assessment of sex-specific differences in fetal development during normal gestation. Sci Rep. 2020;10(1):9399.

76. Segers K, Zhang W, Aourz N, Bongaerts J, Declerck S, Mangelings D, et al. CE-MS metabolic profiling of volumerestricted plasma samples from an acute mouse model for epileptic seizures to discover potentially involved metabolomic features. Talanta. 2020;217:121107.

77. Liao H-W, Rubakhin SS, Philip MC, Sweedler JV. Enhanced single-cell metabolomics by capillary electrophoresis electrospray ionization-mass spectrometry with field amplified sample injection. Anal Chim Acta. 2020;1118:36-43.
78. Xu X, Li L, Weber SG. Electrochemical and optical detectors for capillary and chip separations. Trends Anal Chem. 2007;26(1): 68-79.

79. Lahouidak S, Soriano ML, Salghi R, Zougagh M, Ríos Á. Graphene quantum dots for enhancement of fluorimetric detection coupled to capillary electrophoresis for detection of ofloxacin. Electrophoresis. 2019;40(18-19):2336-41.

80. Prior A, Coliva G, de Jong GJ, Somsen GW. Chiral capillary electrophoresis with UV-excited fluorescence detection for the enantioselective analysis of 9-fluorenylmethoxycarbonylderivatized amino acids. Anal Bioanal Chem. 2018;410(20): 4979-90.

81. Huo F, Wan T, Wang Y, Liu Y, Karmaker PG, Yang X. Enhanced light-emitting diode induced fluorescence detection system with capillary electrophoresis. J Chromatogr A. 2020;1619:460935.

82. Petersen BV, Gallion L, Allbritton NL. Silicon photomultipliers as a low-cost fluorescence detector for capillary electrophoresis. Anal Chem. 2020;92(20):13683-7.

83. Casto LD, Do KB, Baker CA. A miniature 3D printed LEDinduced fluorescence detector for capillary electrophoresis and dual-detector Taylor dispersion analysis. Anal Chem. 2019;91(15):9451-7.

84. Sun S, Wei Y, Wang H, Cao Y, Deng B. A novel electrochemiluminescence sensor coupled with capillary electrophoresis for simultaneous determination of quinapril hydrochloride and its metabolite quinaprilat hydrochloride in human plasma. Talanta. 2018;179:213-20.

85. Gonçalves Silva G, Yamassaki de Almeida E, Seber P, Henrique Settanni P, Pereira de Oliveira A, Ferreira Santos MS, et al. Application of capillary electrophoresis combined with conductometric and UV detection to monitor meteorite simulant bioleaching by Acidithiobacillus ferrooxidans. Electrophoresis. 2018;39(22):2898-2905.

86. Claude B, Cutolo G, Farhat A, Zarafu I, Ionita P, Schuler M, et al. Capillary electrophoresis with dual detection UV/C4D for monitoring myrosinase-mediated hydrolysis of thiol glucosinolate designed for gold nanoparticle conjugation. Anal Chim Acta. 2019; 1085:117-25.

87. Beutner A, Scherer B, Matysik F-M. Dual detection for nonaqueous capillary electrophoresis combining contactless conductivity detection and mass spectrometry. Talanta. 2018;183:33-8.

88. Francisco KJM, do Lago CL. Improving thermal control of capillary electrophoresis with mass spectrometry and capacitively coupled contactless conductivity detection by using 3D printed cartridges. Talanta. 2018;185:37-41.

89. Wang C, Xing H, Zheng B, Yuan H, Xiao D. Simulation and experimental study on doubled-input capacitively coupled contactless conductivity detection of capillary electrophoresis. Sci Rep. 2020;10(1):7944.

90. Domínguez-Vega E, Haselberg R, Iperen Dv, Kool J, Somsen GW, de Jong GJ. Development of a surface plasmon resonance sensor for coupling to capillary electrophoresis allowing affinity assessment of protein mixture components. Sensors Actuators B Chem 2018;254:1040-1047.

91. Kimlinger MJ, Martin RS. The use of a 3D-printed microfluidic device and pressure mobilization for integrating capillary electrophoresis with electrochemical detection. Electroanalysis. 2018;30(10):2241-9.

92. Nowak PM, Wozniakiewicz M. On-line coupling between capillary electrophoresis and microscale thermophoresis (CE-MST); the proof-of-concept. Analyst. 2018;143(20):4854-9.

93. Morani M, Mai TD, Krupova Z, Defrenaix P, Multia E, Riekkola $\mathrm{M}-\mathrm{L}$, et al. Electrokinetic characterization of extracellular vesicles with capillary electrophoresis: a new tool for their identification and quantification. Anal Chim Acta. 2020;1128:42-51. 
94. Furter JS, Boillat M-A, Hauser PC. Low-cost automated capillary electrophoresis instrument assembled from commercially available parts. Electrophoresis. 2020;41(24):2075-82.

95. Le TB, Hauser PC, Pham TNM, Kieu TLP, Le TPQ, Hoang QA, et al. Low-cost and versatile analytical tool with purpose-made capillary electrophoresis coupled to contactless conductivity detection: application to antibiotics quality control in Vietnam. Electrophoresis. 2020;41(23):1980-90.

96. Huge BJ, Champion MM, Dovichi NJ. Capillary zone electrophoresis with fraction collection for separation, culturing, and identification of bacteria from an environmental microbiome. Anal Chem. 2019;91(7):4649-55.

97. Wright SN, Huge BJ, Dovichi NJ. Capillary zone electrophoresis separation and collection of spermatozoa for the forensic analysis of sexual assault evidence. Electrophoresis. 2020;41(15):1344 53.

98. Krenkova J, Liskova M, Cmelik R, Vigh G, Foret F. Multicationic aminopyrene-based labeling tags for oligosaccharide analysis by capillary electrophoresis-mass spectrometry. Anal Chim Acta. 2020;1095:226-32.

99. Mancera-Arteu M, Lleshi N, Sanz-Nebot V, Giménez E, Benavente F. Analysis of glycopeptide biomarkers by on-line $\mathrm{TiO} 2$ solid-phase extraction capillary electrophoresis-mass spectrometry. Talanta. 2020;209:120563.
100. Shao H, Reider B, Jarvas G, Guttman A, Jiang Z, Tran NT, et al. On-line enrichment of $\mathrm{N}$-glycans by immobilized metal-affinity monolith for capillary electrophoresis analysis. Anal Chim Acta. 2020;1134:1-9.

101. Yao H-W, Guo X-F, Wang H. Simultaneous quantitation of intraand extracellular nitric oxide in single macrophage RAW 264.7 cells by capillary electrophoresis with laser-induced fluorescence detection. Anal Chem. 2020;92(17):11904-11.

102. Zhang C, Woolfork AG, Suh K, Ovbude S, Bi C, Elzoeiry M, et al. Clinical and pharmaceutical applications of affinity ligands in capillary electrophoresis: a review. J Pharm Biomed Anal. 2020;177: 112882.

103. Saar-Reismaa P, Brilla C-A, Leiman K, Kaljurand M, Vaher M, Kulp M, et al. Use of a newly-developed portable capillary electrophoresis analyser to detect drugs of abuse in oral fluid: a case study. Talanta. 2020;211:120662.

104. Świądro M, Stelmaszczyk P, Wietecha-Posłuszny R, Dudek D. Development of a new method for drug detection based on a combination of the dried blood spot method and capillary electrophoresis. J Chromatogr B. 2020;1157:122339.

Publisher's note Springer Nature remains neutral with regard to jurisdictional claims in published maps and institutional affiliations. 